\title{
GENERATING DENSE SUBGROUPS OF MEASURE PRESERVING TRANSFORMATIONS
}

\author{
V. S. PRASAD ${ }^{1}$
}

\begin{abstract}
ABSTRACr. Except for a set of first category, all pairs of measure preserving transformations generate a dense subgroup of $G$, the group of all invertible measure preserving transformations of the unit interval when $G$ has the weak topology.
\end{abstract}

Consider $G$ the group of invertible (Lebesgue) measure preserving transformations of the unit interval onto itself. On $G$, we put the weak topology (Halmos [2, pp. 61-67]). It has been asked by A. Iwanik, whether the group generated by certain pairs of measure preserving transformations is dense in $G$. The purpose of this note is to prove: Except for a set of first category in $G \times G$, all pairs of measure preserving transformations generate a subgroup dense in $G$.

Related work has been done by R. Grzaślewicz [1] who showed that for any two positive irrational numbers $\alpha$ and $\beta$, less than 1 , the following transformations $S_{\alpha}$ and $T_{\beta}$ generate a dense subgroup of $G$, where

$$
S_{\alpha}(x)=x+\alpha(\bmod 1)
$$

and

$$
T_{\beta}(x)= \begin{cases}x+\beta\left(\bmod \frac{1}{2}\right), & 0<x<\frac{1}{2}, \\ x, & \frac{1}{2}<x<1 .\end{cases}
$$

It is a classical result of Schreier and Ulam [3] that for a compact connected second countable topological group the pairs of elements generating a dense subgroup is residual $\mathcal{G}_{\delta}$. Note in our case $G$ is not compact.

Preliminary Remarks. We shall follow the terminology used by Halmos [2, pp. 61-67]. A dyadic subbasic neighborhood for $S \in G$ is a set of the form $\{T \in G$ : $m(S D \triangle T D)<\varepsilon\}$, where $D$ is a dyadic set (i.e. a finite union of dyadic intervals), $m$ denotes ordinary Lebesgue measure, $\triangle$ denotes symmetric difference, and $\varepsilon>0$ is a rational number. These sets constitute a subbasis for the weak topology of $G$, and finite intersections of them constitute a basis. With the weak topology $G$ is a complete metrisable group. By a (cyclic) dyadic permutation of rank $n$ in $G$, we mean an invertible measure preserving transformation that maps each dyadic

Received by the editors November 11, 1980.

1980 Mathematics Subject Classification. Primary 28D05.

Key words and phrases. Measure preserving transformation, weak topology, cyclic dyadic permutation.

${ }^{1}$ Supported by a grant from the Natural Sciences and Engineering Research Council Canada (no. U0080). 
interval of rank $n$ onto a dyadic interval of rank $n$ by ordinary translation (in one cycle). The fundamental result is the following Weak Approximation Theorem of Halmos [2, p. 65]. Every dyadic neighborhood contains cyclic permutations of all sufficiently high ranks. Note that we have written "all sufficiently high ranks" instead of "arbitrarily high rank" as in [2]. This slight change is implicit in the proof in [2]. Let $\left\{N_{i}: i=1,2, \ldots\right\}$ be any countable base for $G$ (e.g. dyadic neighborhoods centered at dyadic permutations).

Proof of THE MAIN Result. For each $i=1,2, \ldots$ set

$$
\mathscr{D}_{i}=\left\{(S, T) \in G \times G: \operatorname{gp}\{S, T\} \cap N_{i} \neq \varnothing\right\}
$$

where by $\operatorname{gp}\{S, T\}$ we mean the group generated by $S$ and $T$. It is clear that each $\mathscr{D}_{i}$ is open ( $G$ is a topological group). The set $\mathscr{D}=\bigcap_{i>1} \mathscr{D}_{i}$ consists of all pairs $(S, T)$ such that $\operatorname{gp}\{S, T\}$ is dense in $G$. Since $G \times G$ is a Baire space, we need only show that each $\mathscr{D}_{i}$ is dense in $G \times G$. Any open set in $G \times G$ contains one of the form $N_{j} \times N_{k}$. Thus, to show $\mathscr{D}_{i}$ is dense we must find $(S, T)$ in $N_{j} \times N_{k}$ such that $\operatorname{gp}\{S, T\} \cap N_{i} \neq \varnothing$.

Choose an integer $n$ sufficiently large so that

(1) $P_{i} \in N_{i}, P_{j} \in N_{j}, P_{k} \in N_{k}$ are all cyclic dyadic permutations of rank $n$, and

(2) if we change $P_{k}$ on a set of measure not more than $6 / 2^{n}$, then the resulting transformation will still be in $N_{k}$.

Number the dyadic intervals of rank $n$ in the order that $P_{j}$ permutes them; thus

$$
P_{j}\left(D_{1}\right)=D_{2}, \quad P_{j}\left(D_{2}\right)=D_{3}, \ldots, P_{j}\left(D_{2^{n}-1}\right)=D_{2^{n}} \quad \text { and } \quad P_{j}\left(D_{2^{n}}\right)=D_{1}
$$

where the $D$ 's are the dyadic intervals of rank $n$. Set $S=P_{j}$ and consider $\sigma=\left(1,2, \ldots, 2^{n}\right)$ the corresponding cyclic permutation in $\mathcal{S}_{2^{n}}$, the symmetric group on $2^{n}$ letters.

$P_{k}$ also permutes $D_{1}, D_{2}, \ldots, D_{2^{n}}$ in one cycle and let $\tau=\left(t_{1}, t_{2}, \ldots, t_{2^{n}}\right)$ be the corresponding cyclic permutation in $\mathcal{S}_{2^{n}}$. Consider $\tau^{\prime} \in \mathcal{S}_{2^{n}}$ which differs from $\tau$ only in the following ways

$\tau^{\prime}(1)=2, \tau^{\prime}(2)=1$ and

$\tau^{\prime}$ fixes the symbol $2^{n}$.

Thus if we let $\tau^{\prime}$ be the permutation induced by $\tau$ elsewhere, then it is easy to verify

$$
\left\{t: \tau(t) \neq \tau^{\prime}(t)\right\} \subseteq\left\{1,2, \tau^{-1}(1), \tau^{-1}(2), 2^{n}, \tau^{-1}\left(2^{n}\right)\right\} .
$$

Now, $\tau^{\prime}$ is the product of the transposition $(1,2)$ and a $2^{n}-3$ cycle. Thus $\left(\tau^{\prime}\right)^{2^{n}-3}=(1,2)$. It is a simple exercise in group theory to show that $\mathcal{S}_{2^{n}}$ is generated by the cycle $\sigma=\left(1,2, \ldots, 2^{n}\right)$ and the transposition $(1,2)$. Thus if we set $T$ to be the dyadic permutation of rank $n$ which corresponds to $\tau^{\prime}$, then some word in $S$ and $T$ will permute the dyadic intervals of rank $n$ in exactly the same way as $P_{i} \in N_{i}$. Since $T$ differs from $P_{k}$ on at most 6 dyadic intervals of rank $n$, having total measure not more than $6 / 2^{n}$, then by our choice of $n, T \in N_{k}$. Thus we have shown

THEOREM. Let $G$, the group of invertible measure preserving transformations of the unit interval, have the weak topology. Then the pairs of elements in $G \times G$ which generate a dense subgroup of $G$ forms a dense $\mathcal{G}_{\delta}$ set. 
The author wishes to thank J. Choksi, S. Eigen and the referee for their suggestions.

\section{REFERENCES}

1. R. Grzaślewicz, Density theorems for measurable transformations (preprint).

2. P. R. Halmos, Lectures on ergodic theory, Chelsea, New York, 1956.

3. J. Schreier and S. Ulam, Sur le nombre des générateurs d'un groupe topologique compact et connexe, Fund. Math. 24 (1935), 302-304.

Département de Mathématique, Universtté de Sherbrooke, Shrrbrooke, Québec, Canada J1K 2R1 